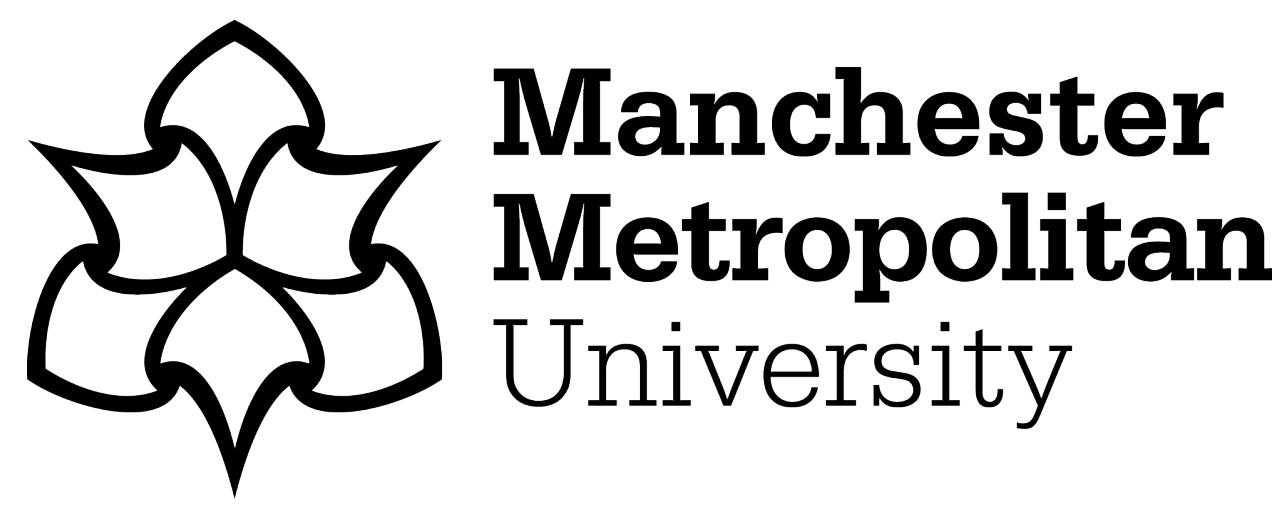

Chen, L, Gao, Z, Liu, B, LV, Y, An, M and Feng., J (2018) Circumferential variation in mechanical characteristics of porcine descending aorta. BIOCELL, 42 (1). pp. 25-34. ISSN 1667-5746

Downloaded from: https://e-space.mmu.ac.uk/622367/

Publisher: Computers, Materials and Continua (Tech Science Press)

DOI: https://doi.org/10.32604/biocell.2018.06114

Usage rights: Creative Commons: Attribution 4.0

Please cite the published version 


\title{
Circumferential variation in mechanical characteristics of porcine descending aorta
}

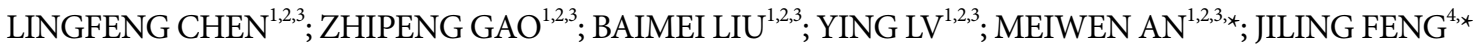 \\ ${ }^{1}$ Institute of Applied Mechanics and Biomedical Engineering, Taiyuan University of Technology, Taiyuan 030024, China \\ ${ }^{2}$ Shanxi Key Laboratory of Material Strength \& Structural Impact, College of Mechanics, Taiyuan University of Technology, Taiyuan 030024, China \\ ${ }^{3}$ National Demonstration Center for Experimental Mechanics Education (Taiyuan university of Technology), Taiyuan 030024, China \\ ${ }^{4}$ School of engineering, Faculty of Science and Engineering, Manchester Metropolitan University, UK
}

Key words: Thoracic aortic aneurysm; Strain-energy function; uniaxial test; elastic modulus; elastin; collagen

\begin{abstract}
Arterial characterization of healthy descending thoracic aorta (DTA) is indispensable in determining stress distributions across wall thickness and different regions that may be responsible for aorta inhomogeneous dilation, rupture, and dissection when aneurysm occurs. Few studies have shown the inhomogeneity of DTA along the aorta tree considering changes in circumferential direction. The present study aims to clarify the circumferential regional characterization of DTA. Porcine DTA tissues were tested according to region and orientation using uniaxial tension. For axial test, results show that the difference in circumferential direction was mainly in collagen fiber modulus, where the anterior collagen fiber modulus was significantly lower than the posterior quadrant. For circumferential test, the difference in circumferential direction was mainly in the recruitment parameter, where the circumferential stiffness is significantly higher in the posterior region at physiological maximum stress. The proximal posterior quadrant and left quadrant showed significantly lower axial collagen fiber stiffness than the right and anterior quadrants, which may be a factor in aneurysm development. Furthermore, the constitutive parameters for similar detailed regions can be used by biomedical engineers to investigate improved therapies and thoroughly understand the initial stage of aneurysm development. The regional collagen fiber modulus can help improve our understanding of the mechanisms of arterial dilation and aortic dissection.
\end{abstract}

\section{Introduction}

Thoracic aortic aneurysm (TAA) is a prevalent and deadly disease with an incidence of approximately 10.4 per 100,000 individuals (Ramanath et al., 2009). It is a silent disease, owing to TAAs normally grow in an indolent manner until dissection or rupture occurs (Elefteriades et al., 2010). The descending thoracic aorta (DTA) has complex geometric, microstructural, and hemodynamic characteristics. Atherosclerosis, aneurysm, and dissection may occur in this part of the aorta. The occurrence, development, and pathological changes of these vascular diseases are closely related to the geometrical morphology and microstructure of blood vessels. The aforementioned diseases also interact with the mechanical properties and hemodynamic characteristics of the vascular wall (Tang and Li, 2016; Tang et al., 2016; Tang and Li, 2018; Yanxia et al. 2017).

${ }^{*}$ Address correspondence to: Meiwen An, meiwen_an@163.com;

Jiling Feng,

j.feng@mmu.ac.uk
Therefore, systematically studying the geometrical morphology and mechanical properties of DTA is necessary for the clinical diagnosis and treatment of aortic disease.

The aorta is composed of intima, media, and adventitia. The aortic passive mechanical behavior is determined mainly by elastin and collagen fibers (Hwl et al., 2018). Previous experimental studies have demonstrated that the arterial tissue is an anisotropic material with nonlinear characteristics. Arterial biomechanics along the axis have been intensively studied. The aortic stiffness is considered to increase away from heart (Han et al., 1995; Kim and Baeck, 2011; Peña et al., 2015). This mechanical behavior change may be due to distal increase in the ratio of collagen to elastin content (Roveri et al., 2010; Sokolis et al., 2008). The wall thickness and diameter also decrease from proximal to distal DTA. Although regional studies for the ascending aorta has been intensively studied (Choudhury et al., 2009; Sokolis et al., 2015; Iliopoulos et al., 2009), few experimental studies have been conducted on circumferential variations in the mechanical behavior of DTA. Kim and Baeck (2011) investigated porcine proximal DTA through inflation test and found that the circumferential stiffness of the posterior 
region was significantly higher than that of the anterior region. Kermani et al. (2016) studied the inhomogeneity of porcine DTA through nanoindentation test and showed that the posterior quadrant is the most compliant quadrant in long indentation. Both these previous studies showed that the aortic wall thickness changed gradually along the circumference, where the anterior region was the thickest and the posterior region was the thinnest. Therefore, systematic mechanical properties and microstructural features of DTA along its circumference need further investigation.

Various tests (e.g. uniaxial and biaxial tensile, inflation, nanoindentation, and non-invasive ultrasonic image tests) have been conducted to study the biomechanics of the arterial wall (Peña et al., 2015; Sokolis et al., 2012); Kim and Baeck, 2011; Kermani et al., 2016; Shcherbakova et al., 2014). However, the results of previous studies were inconsistent probably because of different research methods used, different regions examined, or variations of residual stress. In the present study, we performed an uniaxial extension test to investigate the mechanical behavior of the regional porcine DTA under relatively large loads. Compared with other test methods, uniaxial extension tests perform better in the supra-physiological loading domain. Using uniaxial extension tests, meaningful results can be obtained (Holzapfel et al., 2005). Owing to their simplicity and effectiveness, uniaxial extension tests are widely employed to study relevant mechanical issues (Peña et al., 2015; Qiang et al., 2016).

To clarify the circumferential regional characterization of DTA, porcine DTAs were divided into 16 regions, i.e., 4 axial and 4 circumferential. The maximum physiological stiffness and the stiffness by elastin and collagen were determined through uniaxial tensile tests in both axial and circumferential directions. Finally, Fung-type material parameters for all the regional mean curves were fitted and given. Several conclusions can be drawn from the test results and used as a reference to improve the FEM model of DTA and predict the dilation-prone locations.

\section{Material and Methods}

\section{Specimen collection and initial processing}

Fifteen DTAs of five-month-old pigs were harvested from a local slaughterhouse and immediately stored in an ice-filled box for transport. Among these DTAs, 9 were set aside for the axial test and 6 for the circumferential test. All samples were frozen in physiological solution at $-20^{\circ} \mathrm{C}$ until experimental testing. Excess tissues were removed mechanically from these specimens. To study the differences between regions, the DTAs were divided into 16 parts, i.e. 4 parts for longitudinal/ axial direction: proximal, middle proximal (mid-proximal), middle distal (mid-distal), and distal. Each part was subdivided into four segments: anterior, left, posterior, and right (Fig. 1).

\section{Specimen preparation}

Axial samples were punched by a standard dog-bone model ( $4 \mathrm{~mm}$ wide and $25 \mathrm{~mm}$ long). Circumferential samples with mean width and long of $3.22 \pm 0.29 \mathrm{~mm}$ and $12.35 \pm 1.71 \mathrm{~mm}$ were tested. The thickness of the specimen was measured by a laser displacement sensor (LK-G5000 series, $0.0001 \mathrm{~mm}$ resolution). Two hundred and forty samples with a mean thickness of $1.81 \pm 0.39 \mathrm{~mm}$ were finally tested.

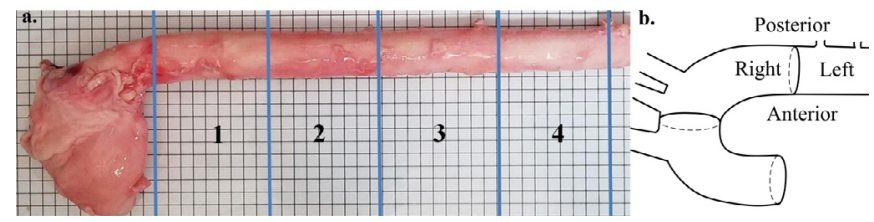

FIGURE 1. Segments of the descending aorta.

\section{Mechanical testing}

An Instron 5544 tester (with a $5 \mathrm{~N}$-load cell) was used to conduct uniaxial tests. The corresponding resolutions of force and displacement were $0.001 \mathrm{~N}$ and $0.001 \mathrm{~mm}$, respectively. Prior to the test, sandpaper was glued to the ends of clamps to prevent slippage. The axial strains of dog-bone-shaped samples were measured using a digital image correlation system equipped with a high-performance digital camera (Fujinon HF25HA-1B, 1.5 million pixels). The axial strains of the rectangular samples were measured using a built-in displacement sensor. An ultrasonic humidifier was used to keep the specimens wet during the test. Following previous research (Peña et al., 2015), we assumed that the axial prestretch of the proximal and distal thoracic aorta were 1.21 and 1.43 , respectively. To eliminate the hysteresis effect of biological tissues and obtain the repeatable stress-strain curves, the tests were applied by preliminary cycles under quasi-static conditions with $3 \mathrm{~mm} / \mathrm{min}$ displacement rate. The proximal and distal specimens were preconditioned for 4 cycles at $21 \%$ and $43 \%$ stretch, respectively. The midproximal and mid-distal specimens were preconditioned for 4 cycles at the mean load for each specimen. The mean load was calculated by the measurement results under 0.21 stretch of the proximal specimen and 0.43 stretch of the distal specimen. All the circumferential specimens were preconditioned at 0.1 $\mathrm{MPa}$ stress for 4 times. Following preconditioning, another loading (with $3 \mathrm{~mm} / \mathrm{min}$ displacement rate) was conducted to rupture the specimens or reach the limit load.

The Cauchy stresses were determined as

$$
\begin{aligned}
& \sigma_{\theta \theta}=\left(\text { Force }_{\theta} * \lambda_{\theta}\right) /\left(\text { thickness }_{z} * \text { width }_{z}\right), \\
& \sigma_{z z}=\left(\text { Force }_{z} * \lambda_{z}\right) /\left(\text { thickness }_{\theta} * \text { width }_{\theta}\right)
\end{aligned}
$$

where $\theta$ and $z$ mean circumferential and axial directions. Force is the load registered by the Instron machine. Thickness and width are the initial thickness and width, respectively, while $\lambda$ is the stretch ratio. To minimize the impact of thickness on testing, we chose the area with small thickness change as the research object. When measuring the thickness, the average thickness is calculated by the selected effective measuring area of the specimen. The thickness of the clamped part did not participate in the calculation of stress.

\section{Data analysis}

To study the stress-stretch curves, including high-stress range, we considered a mathematical model introduced by Raghavan et al. 1996. The relationship between stretch and stress can be expressed as

$\varepsilon=\lambda-1=\left(K+\frac{A}{B+\sigma}\right) \sigma$ 
where $\varepsilon$ is the strain, $\lambda$ is the stretch ratio, $\sigma$ is the stress resulting from the applied uniaxial tension; and $K, A$, and $B$ are model parameters. $K$ is the reverse of the maximum modulus of the tissue, $A$ is the recruitment parameter, and $B$ is the value of stress at the intersection of the lines defined by initial and maximum moduli. According to this theory, the aorta can simply be considered as containing two primary load-bearing fibers: elastin and collagen. Only the elastin fibers are taut in the low loading domain (see Fig. 2, phase 1). As $\sigma \rightarrow 0$ and $B+\sigma \approx B$, Young's modulus of elastin $\left(E_{E}\right)$ is written from Eq. (1) in the following form:

$$
E_{E}=\left.\frac{d \sigma}{d \varepsilon}\right|_{\sigma \rightarrow 0}=\frac{1}{\left(K+\frac{A}{B}\right)}
$$

The slope of the stress-strain curve increases gradually as collagen fibers start contributing to the load bearing when the tissue is stretched (see Fig. 2, phase 2). All collagen fibers are considered recruited into the load bearing at a certain point prior to tissue failure. The slope of the final portion of the stress-strain curve corresponds to the combined total stiffness of the elastin and collagen fibers (see Fig. 2,

phase 3). We assume that $B<<\sigma_{y}$ during phase 3 , so that $B+\sigma \approx \sigma$, and according to Eq. (1), $E_{C}$ can be written as

$E_{c}=\frac{1}{K}-\frac{1}{K+\frac{A}{B}}=\frac{A}{K(A+K B)}$

For detail: $K$ is the inverse of $\left(E_{\text {elastin }}+E_{\text {collagen }}\right)$. This means that $K$ is the inverse of the combined contribution to total tissue stiffness by the elastin and collagen fibers. $A$ is the strain intercept of the final portion (phase 3 ) of the stressstrain curve (see Fig. 2). As shown by Raghavan et al. (1996), $A$ is inversely proportional to the rate of recruitment of the collagen fibers for a given strain rate, or directly proportional to the average degree of tortuosity of the collagen fibers in the tissue. $A$ can be assume as the "recruitment parameter", the smaller the $A$, the faster the collagen fibers activate. $B$ is the value of stress at the intersection of the lines defined by the linear responses in phase 1 and phase 3 (dashed and solid lines, respectively, see Fig. 2)

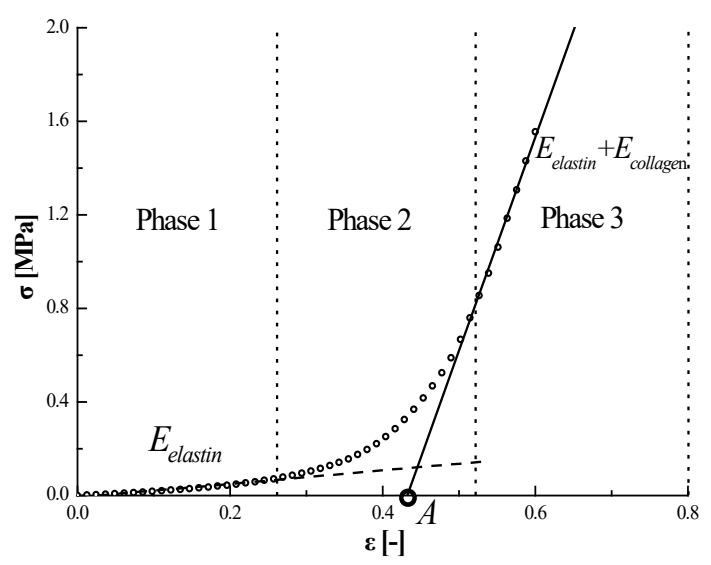

FIGURE 2. An example demonstrates the relationship between elastin fiber modulus, collagen fiber modulus and tress-strain curve.
The maximum modulus at physiological limits was also considered. The stiffness at $0.1 \mathrm{MPa}$ (Sokolis 2007) was calculated as tangent modulus:

Stiffness $_{0.1 M P a}=\left.\frac{\partial \sigma}{\partial \lambda}\right|_{\sigma=0.1 M P a}$

Furthermore, we considered the Fung-type strain energy function (SEF) for describing the regional characters (Fung et al., 1993). The mean stress-stretch curves were fitted, assumed that the material is incompressible, homogeneous, nonlinear, and anisotropic:

$$
W=K^{1}\left(e^{Q}-1\right), \quad Q=c_{\theta \theta} E_{\theta}^{2}+c_{z z} E_{z}^{2}+c_{\theta z} E_{\theta} E_{z}
$$$$
\text { where } E_{i}=1 / 2\left(\lambda_{i}^{2}-1\right), \quad i=\theta, z \text { where the }
$$
circumferential and axial Green strains. Material constant $K^{1}$ (stress units, the superscript 1 for distinguishing the former

$K)$ served as scaling factor, whereas constants $c_{\theta \theta}, c_{z z}$, and $c_{\theta z}$ (unitless) denoted circumferential stiffness, axial stiffness, and stiffness interaction between the two axes. The Cauchy stress were defined as

$$
\sigma_{i}=\lambda_{i}^{2} \frac{\partial W}{\partial E_{i}}
$$

For this SEF, the specific fitting method and the calculation method for root-mean-square error $\varepsilon$ and determination coefficient were followed by Iliopoulos et al. (2013). The fitting method was validated by Sokolis et al., 2012. The fitting of the experimental data was developed by using a Levenberg-Marquardt type minimization algorithm. $\varepsilon \leq 0.1$ is representing a good fit to the experimental data. In this study, data under the 1.6 stretch for axial specimens and under the 1.8 stretch for circumferential specimens were used in the analysis.

Results are given as mean \pm standard deviation (SD). The groups were compared by one-way ANOVA followed by Turkey test and multiple comparisons followed by Newman-Keuls multiple comparisons test using SPSS v17.0 (SPSS Inc). $p<0.05$ was considered statistically significant.

\section{Results}

\section{Inner diameter and thickness}

The mean thickness and inner diameter decrease away from the heart (Fig. 3). The statistical analysis revealed significant differences between the values of proximal DTA and distal DTA $(p<0.01)$. The wall thickness changed gradually along the circumference (Fig. 3b). The anterior wall was the thickest and the posterior wall was the thinnest.

\section{Mechanical testing and data fitting}

The axial and circumferential mean stress-stretch curves for the four circumferential regions along the aorta are shown in Figs. 4 and 5, respectively. All the tissues exhibit a pronounced nonlinear mechanical response. In the lowstress range, the stress-stretch response for each region 
was nearly linear, and the response became nonlinear in the higher-stress range before becoming nearly linear again. For the axial test, the tissues showed non-uniform response at the four circumferential regions. For the circumferential test,

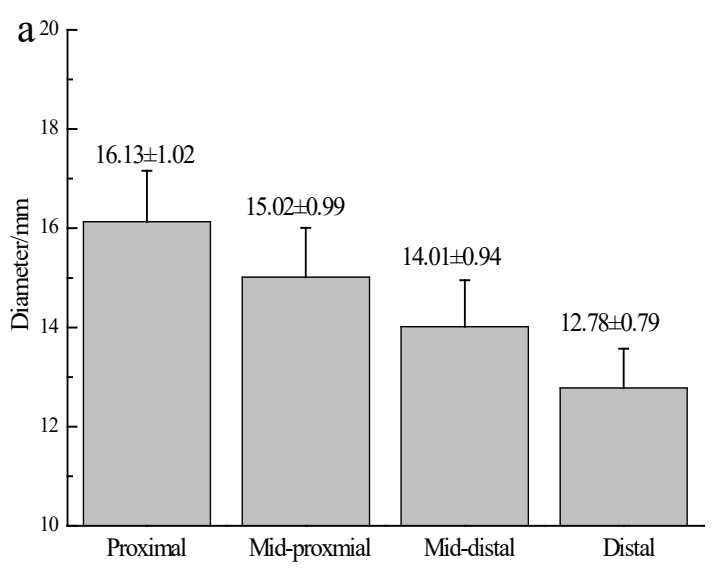

the tissues indicated a relatively regular response in that posterior tensile curves always rise faster than others after the initial linear part.

FIGURE 3. The geometry of the four axial DTA regions: (a) inner diameter, (b) the thickness of the 12 quadrants. Symbol ${ }^{* *}$ denotes $p<0.01$ against anterior aorta and symbol $\dagger \dagger$ denotes $p<0.01$ against posterior aorta, respectively.
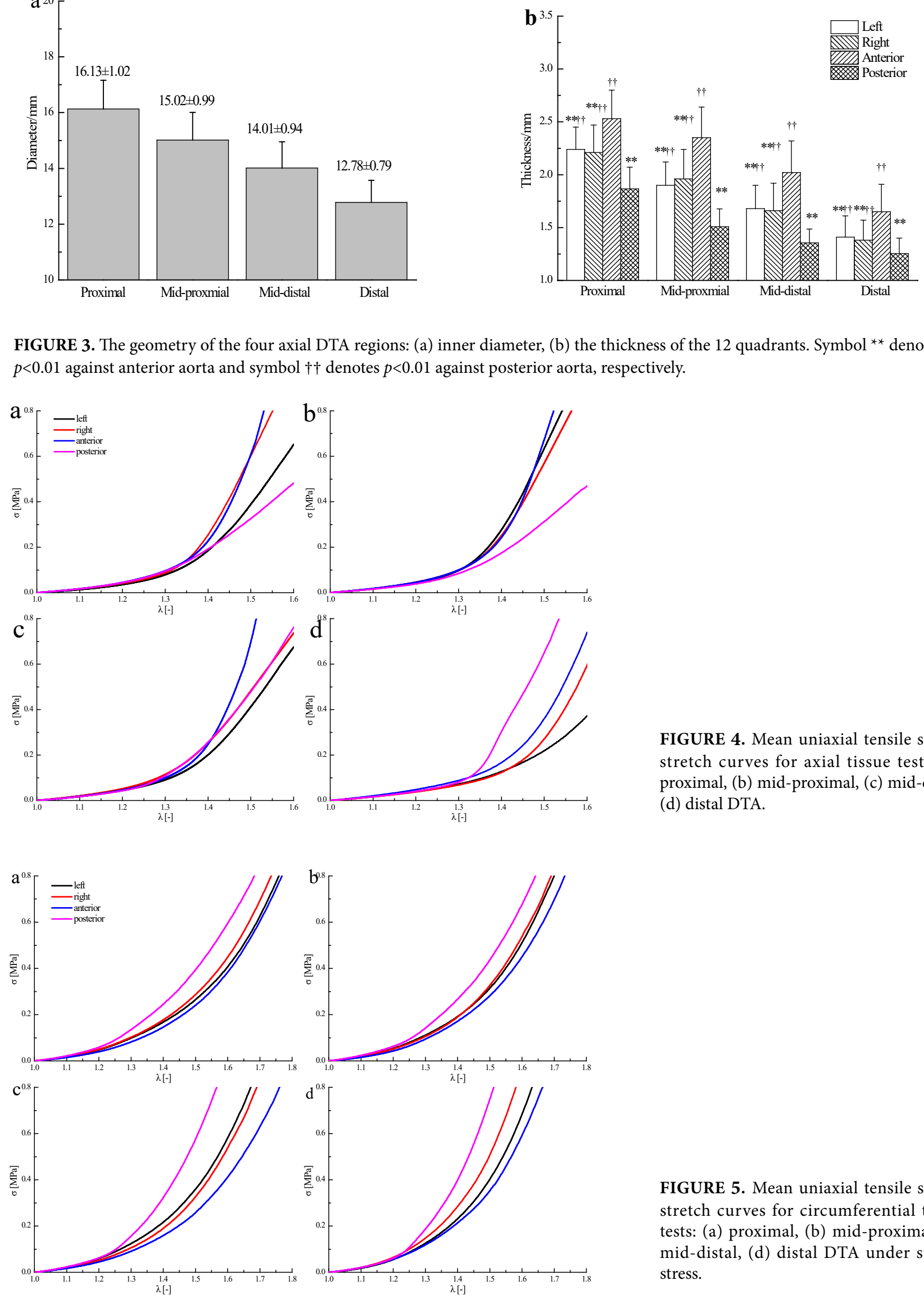

FIGURE 4. Mean uniaxial tensile stressstretch curves for axial tissue tests: (a) proximal, (b) mid-proximal, (c) mid-distal, (d) distal DTA.

FIGURE 5. Mean uniaxial tensile stressstretch curves for circumferential tissue tests: (a) proximal, (b) mid-proximal, (c) mid-distal, (d) distal DTA under suprastress. 
The stress-stretch curves for all the samples showed a satisfactory fit with the mathematical model, indicating a high determination coefficient (Tab. 1). For the axial tissues, the difference was in parameter $\mathrm{K}$ except in the distal region. For the circumferential tissues, the difference was in parameter A. However, the four quadrants showed no significant difference at the mid-proximal region in the circumferential test. The results indicate the difference between the characteristics of the axial and circumferential directions.

The calculated elastin and collagen fiber modulus and the stiffness at $0.1 \mathrm{MPa}$ for the axial and circumferential tissues are shown in Figs. 6 and 7, respectively. For the axial tissues, the anterior collagen fiber modulus showed a significantly lower trend than that of the posterior quadrant at all the axial regions. The left quadrant also exhibited a significantly lower Ecollagen than the anterior and right quadrants at the proximal region. The distal posterior quadrant showed a special characteristic, which has a significantly lower Eelastin and a significantly higher stiffness at $0.1 \mathrm{MPa}$, than other quadrants. For circumferential tissues, the stiffness at $0.1 \mathrm{MPa}$ for the posterior regions were always significantly higher than that of the other quadrants except at the mid-proximal region.

TABLE 1

Mathematical parameters obtained for the corresponding 16 regions curves

\begin{tabular}{|c|c|c|c|c|c|c|}
\hline & \multicolumn{3}{|l|}{ Axial-Specimens } & \multicolumn{3}{|c|}{ Circumferential-Specimens } \\
\hline & $K$ & $A$ & $B$ & $\bar{K}$ & $A$ & $B$ \\
\hline Proximal & & & & & & \\
\hline $\begin{array}{l}\text { Anterior } \\
n=9,5\end{array}$ & $0.141 \pm 0.082$ & $0.458 \pm 0.047$ & $0.054 \pm 0.007$ & $0.266 \pm 0.123$ & $0.659 \pm 0.050$ & $0.113 \pm 0.029$ \\
\hline Left $n=7,5$ & $0.291 \pm 0.089^{* *}$ & $0.447 \pm 0.059$ & $0.047 \pm 0.010$ & $0.205 \pm 0.080$ & $0.696 \pm 0.065$ & $0.148 \pm .0270$ \\
\hline Posterior & $0.508 \pm 0.140^{* * \S \varsigma}$ & $0.407 \pm 0.095$ & $0.056 \pm 0.026$ & $0.313 \pm 0.036$ & $0.504 \pm 0.062^{* * \S \varsigma}$ & $0.118 \pm 0.024$ \\
\hline $\begin{array}{l}n=7,5 \\
\text { Right } n=9,6 \\
\text { Mid-proximal }\end{array}$ & $0.170 \pm 0.045^{\S \S \dagger \dagger}$ & $0.444 \pm .0076$ & $0.047 \pm 0.014$ & $0.243 \pm 0.080$ & $0.638 \pm 0.077^{\dagger \dagger}$ & $0.134 \pm 0.035$ \\
\hline Ant $n=8,5$ & $0.147 \pm 0.035$ & $0.438 \pm 0.020$ & $0.052 \pm 0.066$ & $0.223 \pm 0.058$ & $0.645 \pm 0.101$ & $0.126 \pm 0.044$ \\
\hline Left $n=8,6$ & $0.199 \pm 0.055^{* \star}$ & $0.411 \pm 0.025$ & $0.047 \pm 0.005$ & $0.210 \pm 0.099$ & $0.628 \pm 0.110$ & $0.137 \pm 0.045$ \\
\hline $\begin{array}{l}\text { Posterior } \\
n=7,5\end{array}$ & $0.495 \pm 0.125^{* \star \S \S}$ & $0.434 \pm 0.095$ & $0.052 \pm 0.016$ & $0.206 \pm 0.010$ & $0.560 \pm 0.015$ & $0.144 \pm 0.015$ \\
\hline $\begin{array}{l}\text { Right } n=6,5 \\
\text { Mid-distal }\end{array}$ & $0.196 \pm 0.074^{\dagger \dagger}$ & $0.431 \pm 0.034$ & $0.048 \pm 0.022$ & $0.161 \pm 0.040$ & $0.676 \pm 0.099$ & $0.156 \pm 0.055$ \\
\hline $\begin{array}{l}\text { Anterior } \\
n=9,5\end{array}$ & $0.140 \pm 0.036$ & $0.444 \pm 0.027$ & $0.055 \pm 0.008$ & $0.211 \pm 0.046$ & $0.695 \pm 0.052$ & $0.138 \pm 0.023$ \\
\hline Left $n=8,6$ & $0.264 \pm 0.087^{*}$ & $0.453 \pm 0.030$ & $0.060 \pm 0.008$ & $0.161 \pm 0.030$ & $0.667 \pm 0.075$ & $0.169 \pm 0.050$ \\
\hline Posterior & $0.357 \pm 0.133^{* * \$ S \varsigma}$ & $0.380 \pm 0.071$ & $0.045 \pm 0.015$ & $0.192 \pm 0.034$ & $0.455 \pm 0.048^{* \star \varsigma \S}$ & $0.107 \pm 0.026$ \\
\hline $\begin{array}{l}n=7,5 \\
\text { Right } n=8,5 \\
\text { Distal }\end{array}$ & $0.243 \pm 0.036^{* \dagger}$ & $0.437 \pm 0.052$ & $0.067 \pm 0.012^{* \dagger}$ & $0.161 \pm 0.040$ & $0.675 \pm 0.099^{\dagger \dagger}$ & $0.156 \pm 0.055$ \\
\hline $\begin{array}{l}\text { Anterior } \\
n=9,5\end{array}$ & $0.149 \pm 0.047$ & $0.552 \pm 0.050$ & $0.080 \pm 0.015$ & $0.171 \pm 0.054$ & $0.589 \pm 0.150$ & $0.158 \pm 0.046$ \\
\hline Left $n=6,6$ & $0.253 \pm 0.035$ & $0.638 \pm 0.060$ & $0.09 \pm 0.008$ & $0.156 \pm 0.036$ & $0.611 \pm 0.060$ & $0.143 \pm 0.026$ \\
\hline $\begin{array}{l}\text { Posterior } \\
n=7,5\end{array}$ & $0.251 \pm 0.123$ & $0.365 \pm 0.053^{* * \S s}$ & $0.031 \pm 0.013^{* * \$ s}$ & $0.176 \pm 0.042$ & $0.411 \pm 0.057^{* \$ S}$ & $0.088 \pm 0.022$ \\
\hline Right $n=7,5$ & $0.173 \pm 0.07$ & $0.590 \pm 0.100^{\dagger \dagger}$ & $0.069 \pm 0.022^{\dagger \dagger}$ & $0.138 \pm 0.059$ & $0.590 \pm 0.130^{\dagger}$ & $0.153 \pm 0.044$ \\
\hline
\end{tabular}

Results are presented as mean \pm standard error (SE). $K, A$ and $B$ are material parameters, and $n$ are number of samples. Symbol ${ }^{\star} / *$ denotes $p<0.05 / p<0.01$ against anterior aorta, respectively. Symbol $\S / \S \S$ denotes $p<0.05 / p<0.01$ against left aorta, respectively. Symbol $\dagger / \dagger \dagger$ denotes $p<0.01$ against posterior aorta, respectively. 

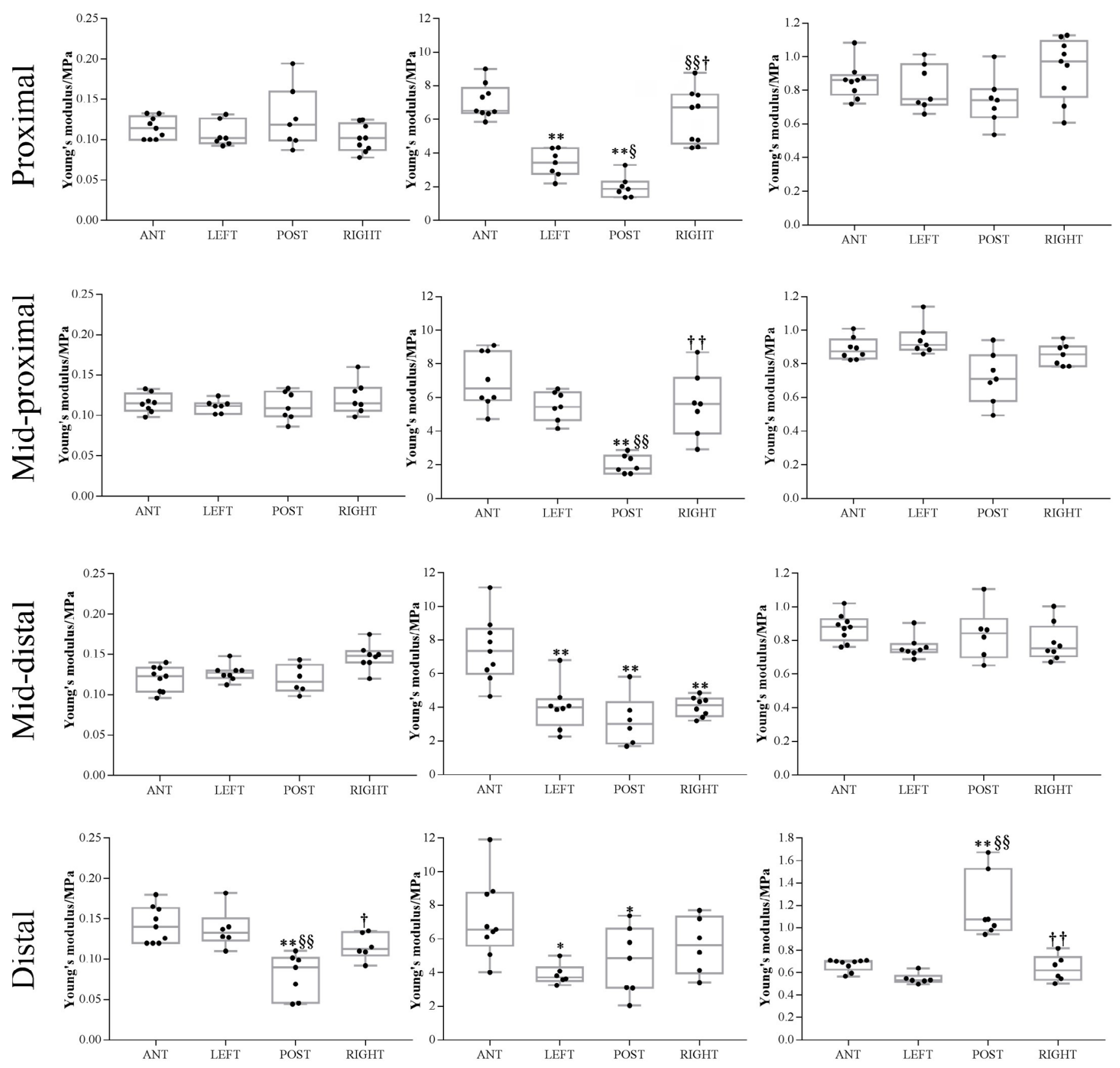

Elastin fiber modulus

Collagen fiber modulus

Stiffness at $0.1 \mathrm{MPa}$

FIGURE 6. Elastin fiber modulus $\left(E_{\text {elastin }}\right)$ and collagen fiber modulus $\left(E_{\text {collagen }}\right)$ and the stiffness at $0.1 \mathrm{MPa}($ Stiffness $)$ for the axial tissues.

Symbol ${ }^{* * * *}$ denotes $p<0.05 / p<0.01$ against anterior aorta, respectively. Symbol $\S / \S \S$ denotes $p<0.05 / p<0.01$ against left aorta, respectively. Symbol $\uparrow / \uparrow \dagger$ denotes $p<0.01$ against posterior aorta, respectively. Symbol $\$ / \$ \S$ denotes $\mathrm{p}<0.05 / \mathrm{p}<0.01$ against left aorta, respectively. Symbol $\dagger / \dagger \dagger$ denotes $\mathrm{p}<0.01$ against posterior aorta, respectively. 

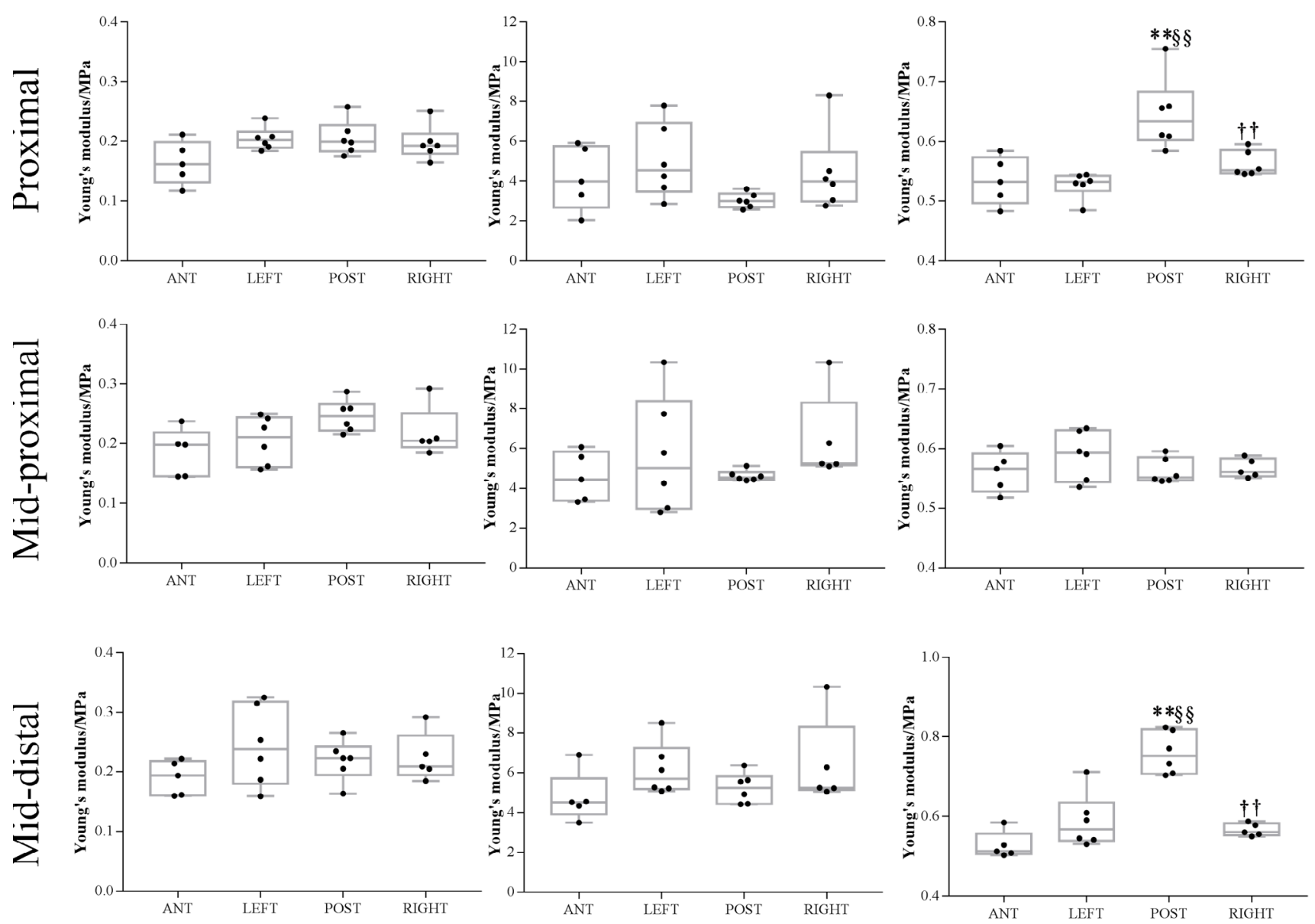

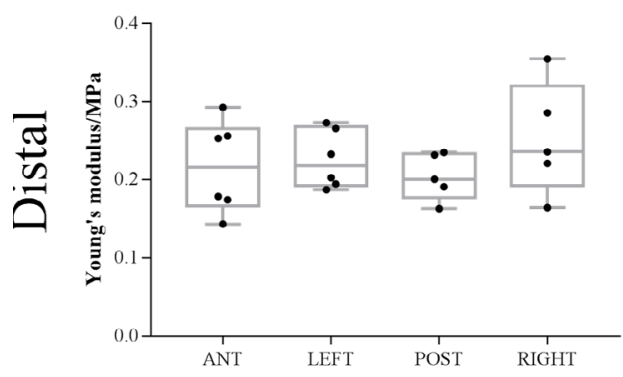

Elastin fiber modulus

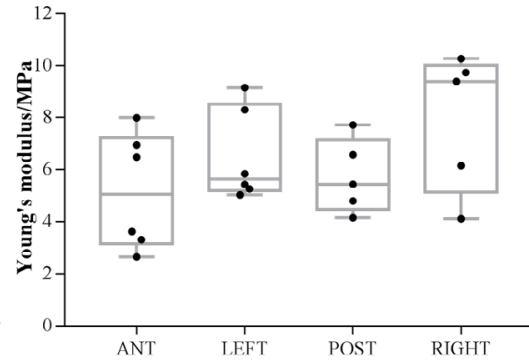

Collagen fiber modulus

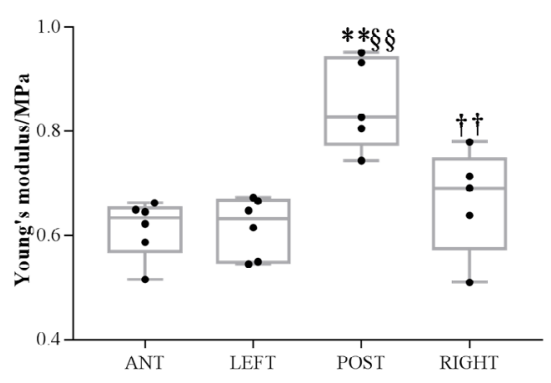

Stiffness at $0.1 \mathrm{MPa}$

FIGURE 7. Elastin fiber modulus $\left(E_{\text {elastin }}\right)$ and collagen fiber modulus $\left(E_{\text {collagen }}\right)$ and the stiffness at $0.1 \mathrm{MPa}\left(\right.$ Stiffness $\left._{0.1 \mathrm{MPa}}\right)$ for the circumferential tissues. Symbol ${ }^{*}{ }^{* *}$ denotes $p<0.05 / p<0.01$ against anterior aorta, respectively. Symbol $\$ / \$ \S$ denotes $p<0.05 / p<0.01$ against left aorta, respectively. Symbol $\dagger / \uparrow \dagger$ denotes $p<0.01$ against posterior aorta, respectively. 
TABLE 2

Material constants obtained from the mean stress-stretch curves of regrouped tissues

\begin{tabular}{|c|c|c|c|c|c|c|}
\hline & $K^{I}(\mathrm{MPa})$ & $c_{\theta \theta}(-)$ & $c_{z z}(-)$ & $c_{\theta z}(-)$ & $\varepsilon(-)$ & $R^{2}$ \\
\hline \multicolumn{7}{|l|}{ Proximal } \\
\hline Anterior & 0.075 & 3.596 & 6.570 & 8.710 & 0.030 & 0.991 \\
\hline Left & 0.133 & 0.542 & 0.718 & 0.0793 & 0.024 & 0.994 \\
\hline Posterior & 0.557 & 0.221 & 0.180 & 0.0425 & 0.017 & 0.998 \\
\hline Right & 0.087 & 2.305 & 4.154 & 5.124 & 0.044 & 0.986 \\
\hline \multicolumn{7}{|l|}{ Mid-proximal } \\
\hline Anterior & 0.088 & 3.056 & 5.511 & 7.171 & 0.036 & 0.991 \\
\hline Left & 0.115 & 3.290 & 5.616 & 7.682 & 0.039 & 0.991 \\
\hline Posterior & 0.349 & 1.493 & 0.995 & 2.112 & 0.020 & 0.998 \\
\hline Right & 0.106 & 3.380 & 5.276 & 7.496 & 0.037 & 0.992 \\
\hline \multicolumn{7}{|l|}{ Mid-distal } \\
\hline Anterior & 0.065 & 2.941 & 6.272 & 7.314 & 0.039 & 0.990 \\
\hline Left & 0.176 & 2.593 & 2.978 & 4.939 & 0.025 & 0.996 \\
\hline Posterior & 0.528 & 1.466 & 1.065 & 2.180 & 0.047 & 0.994 \\
\hline Right & 0.143 & 2.547 & 3.556 & 5.262 & 0.022 & 0.997 \\
\hline \multicolumn{7}{|l|}{ Distal } \\
\hline Anterior & 0.258 & 3.353 & 2.959 & 5.828 & 0.036 & 0.994 \\
\hline Left & 0.152 & 2.020 & 2.811 & 3.908 & 0.043 & 0.992 \\
\hline Posterior & 0.681 & 1.675 & 1.350 & 2.690 & 0.081 & 0.988 \\
\hline Right & 0.233 & 3.067 & 1.830 & 4.275 & 0.030 & 0.997 \\
\hline
\end{tabular}

The Fung-type model provided reasonably accurate fits with all the mean curves with errors $\varepsilon \sim 0.036$ and $\mathrm{R}^{2} \sim 0.993$. Tab. 2 lists the material parameters of mean curves according to region. The results indicate both the axial and circumferential information. Data provided in this study were used as a reference for biomechanical engineers to optimize finite element modeling.

\section{Discussion}

In the present study, the circumferential variation in the mechanical properties of the porcine descending thoracic aorta was experimentally investigated. Through the uniaxial test, the circumferential and axial characteristics of four circumferential quadrants along the descending aorta were analyzed. Compared with existing data that were primarily obtained through inflation and nanoindentation tests, the spatial variations (16 regions) of the material properties were substantially improved. Simplified elastin and collagen fiber modulus and the maximum stiffness of the physiological range were calculated, where the collagen fiber modulus was difficult to obtain in inflation and biaxial experiments for young porcine due to the limited stretch range.

\section{The passive mechanical behavior and anisotropic response}

Similar to other studies (Peña et al., 2015; Pandit et al., 2005; Wang et al., 2006), all regions of the DTA exhibited similar nonlinear behavior (Figs. 4 and 5). Passive biomechanical properties of the aortic wall are determined mainly by elastin and collagen fibers. Generally, the linear behavior of the tissue in the low-pressure range is defined by the elastin fibers (Gundiah et al., 2007), and the non-linear behavior in the high-pressure range is defined by the recruited collagen fibers and the density of cross-linking (Shadwick et al., 1999). The orientation of collagen fibers endows the aortic wall with an anisotropic material response. Our findings demonstrate that the circumferential tissue of the porcine thoracic aorta was significantly stiffer than the axial tissue at the low stress range (Fig. 6 and Fig. 7), which is consistent with previous studies (Peña et al., 2015). This trend is due to the elastin fiber network more toward to circumferential direction (Weisbecker et al., 2013). The stretch-stress curves for the circumferential tissue is regular, i.e. posterior $>$ right $\approx$ left $>$ anterior, which caused by the preferable distribution of the collagen fiber or elastin corresponding to the stress distribution. However, the curves for the axial tissues are not regular. As we known, the axial in situ stretch is dependent on arterial location in the aorta (Han et al., 1995; Peña et al., 2015), and individuals have different intercostal arteries distribute form. These conditions may cause different axially stress distribution.

\section{Circumferential variation of axial $E_{\text {collagen }}$}

To the best of our knowledge, the significant circumferential variation of the stiffness of axial collagen fibers along the aorta has not been reported. The results show that the highest value always appears in the anterior region and the lowest always appears in the posterior region (except the distal region) (Figs. 6 and 7). In this paper, the biological factors affecting vascular growth are not considered. The reduced stiffness of the posterior longitudinally may be due to the effect of in vivo environment, i.e., the stress-growth relationship that the posterior of aorta was fixed by the intercostal arteries and the lower axial stress caused lower collagen and elastin orientate in axial direction. The thickness also affected by the stress growth relationship. Consist with Thubrikar and Kim, in the normal aorta, the wall thickness was highest in the anterior region and gradually decreased toward the posterior region 
(Fig. 3b) (Thubrikar et al., 2001; Kim and Baeck, 2011). However, as the spine support and the dilation induces a simultaneous thinning of the aneurysm wall, the thickness distribution in the aneurysm region appears to be opposite.

In proximal DTA, besides the posterior quadrant, the left quadrant also showed significantly lower axial collagen fiber stiffness than the other two quadrants (Fig. 6). This result is in agreement with the findings of Sokolis et al., (2015) that the maximal axial modulus of the medial and left quadrants is lower than that of the other two quadrants, where the medial quadrant of the ascending aorta is reinforced by the builtin pulmonary artery. This left quadrant axial softer trend at the proximal region may be due to the effect of the following secondary flow. Consequently, the left region tends to expand when the high transmural pressure occurs and aneurysms are more likely to occur in this area. This prediction was validated by the clinical data on 37 Stanford type A and 15 Stanford type B patients among 155 patients (Howard, Banerjee and Fairhead 2013).

\section{Circumferential variation of axial $E_{\text {elastin }}$}

The axial elastin modulus showed no significant difference between the four quadrants except at the distal region. However, Kermani et al., (2016) analyzed the region-specific axial toe modulus using the nanoindentation test and found the right quadrant is the most compliant part in axial indentation. Compared with these findings, the differences of the present study may be due to the scale of measurement, i.e., the passive response of the macro structure is affected by elastin and collagen fibers with their density, crosslinking, and orientation and the nanoindentation response of the micro structure may be affected by the local fiber arrangement.

\section{Circumferential variation of circumferential tissues}

The present study shows that the circumferential stiffness is significantly higher in the posterior region at physiological maximum stress (except in the mid-proximal region, as shown in Fig. 7). This difference is due to the faster response speed of collagen fibers (see the significantly lower A in Tab. 1), where the elastin and collagen fiber modulus along the circumferential quadrants show no statistical difference (Fig. 7; Tab. 1). The four quadrants at the mid-proximal region exhibited a similar passive response. Using inflation test, Kim and Baeck (2011) showed that the posterior region of the upper-half porcine DTA was significantly stiffer than the anterior in the circumferential direction, which is largely consistent with our findings. Interestingly, the distal posterior region showed significantly higher stiffness at physiological maximum stress in both directions. This phenomenon was also noted by Kim and Baeck (2011). These characteristics and associated parameters are valuable references for biomedical engineers working on the design of vascular stents or on the development of improved treatment techniques.

\section{SEF material constants}

The porcine aorta is used as a model of the human aorta in various fields of cardiovascular research, and the young porcine thoracic aorta has shown good correspondence with human tissue aged $<60$ years ( $\mathrm{Hwl}$ et al., 2018). In this study, the porcine descending thoracic aorta was assumed to represent a young and healthy human aorta. The mean curves of each region were fitted. The corresponding Fung-type SEF material constants, as shown in Tab. 1, can serve as input data used by biomedical engineers in investigating advanced therapies and for advanced realistic computational modeling study.

\section{Limitations}

The findings of this study should be interpreted within the context of its limitations. In this study, we have assumed that the aortic wall is materially homogeneous although it has three distinct layers. Furthermore, a Fung-type strainenergy function was used to fit the mean curves, which requires further study on layer-specific mechanical tests and micro-structured constitutive models (Weisbeker et al. 2015). Moreover, at the low stretch ratio of the stress-stretch curve, the smooth muscle and the elastin play a dominate role, and the contribution result from the unfolding of the crimpled elastin/collagen and the rotation of cross-linked elastin/collagen (Gasser et al., 2006; Chen et al., 2016). At the high stretch ratio, the mechanical properties mainly rely on the tension of the after-straightened elastin/collagen. In this paper, the mechanism was simplified, where the collagen modulus was obtained using combined modulus directly minus the elastin modulus. And $B+\sigma$ was simplified using $B+\sigma \approx \sigma$. Finally, we assumed that the mean curves represented the passive response of the porcine thoracic aorta. However, the circumferential and axial data were obtained from different individuals.

\section{Conclusion}

In this study, uniaxially tensile experiments were conducted to test the mechanical properties of porcine DTA in the axial and circumferential directions. The porcine DTA were intensively studied, i.e. DTA were divided into 16 regions for tests. Fitted by a mathematical model, the difference in the axial and circumferential directions was given. The elastin and collagen fiber modulus and the stiffness at $0.1 \mathrm{MPa}$ were also analyzed. Finally, Fung-type SEF material constants were fitted using the mean curves for further study. Compared with previous works, the present study systematically demonstrates the circumferential regional characterization of DTA and provides a possible reason for aneurysm development. Thus, the findings of this study may be a useful reference in biomedical engineering.

\section{Conflict of interest statement: None.}

\section{Acknowledgement}

The authors gratefully acknowledge the support of the Nature Science Foundation of China (Grant No. 11372208; 11502157) and Shanxi Province Programs for Science and Technology Innovation in Colleges and Universities (Grant No. 2016BY062). 


\section{References}

Chen Q, Wang Y, Li ZY (2016). Re-examination of the mechanical anisotropy of porcine thoracic aorta by uniaxial tensile tests. Biomedical Engineering Online 15: 493-506.

Choudhury N, Bouchot O, Rouleau L, Tremblay D, Cartier R, Butany J (2009). Local mechanical and structural properties of healthy and diseased human ascending aorta tissue. Cardiovascular Pathology 18: 83-91.

Elefteriades JA, Farkas EA (2010). Thoracic aortic aneurysm clinically pertinent controversies and uncertainties. Journal of the American College of Cardiology 55: 841-857.

Elizabeth PT, Juan MC, Luis EGQ, María CGR, Roberto O (2017). Effect of synthesis variables of plasma synthesized polymers on growth of HepG2 cells. Biocell 41: 41-43.

Fung YC (1993). Biomechanics: Mechanical Properties of Living Tissues. Springer Press.

Howard DPJ, Banerjee A, Fairhead JF, Perkins J, Silver LE, Rothwell PM (2013). Population-based study of incidence and outcome of acute aortic dissection and premorbid risk factor control 10-year results from the oxford vascular study. Journal of Vascular Surgery 58: 1423-1423.

Gasser TC, Ogden RW, Holzapfel GA (2006). Hyperelastic Modelling of Arterial Layers with Distributed Collagen Fibre Orientations. Journal of the Royal Society Interface 3: 15-35.

Gundiah N, Ratcliffe M (2007). Determination of strain energy function for arterial elastin: experiments using histology and mechanical tests. Journal of Biomechanics 40: 586-594.

Han HC, Fung YC (1995). Longitudinal strain of canine and porcine aortas. Journal of Biomechanics 28: 637-641.

Hwl DB, Ferrara A, Conti M, Moll F L, Herwaarden JA, Figueroa CA (2018). Comparative analysis of porcine and human thoracic aortic stiffness. European Journal of Vascular and Endovascular Surgery 55: 560-566.

Iliopoulos DC, Deveja RP, Kritharis EP, Perrea D, Sionis GD, Toutouzas K (2009). Regional and directional variations in the mechanical properties of ascending thoracic aortic aneurysms. Medical Engineering \& Physics 31: 1-9.

Holzapfel GA, Sommer G, Gasser CT, Regitnig P (2005). Determination of layer-specific mechanical properties of human coronary arteries with nonatherosclerotic intimal thickening and related constitutive modeling. American Journal of Physiology Heart \& Circulatory Physiology 289 . 2048-2058.

Kermani G, Hemmasizadeh A, Assari S, Autieri M, Darvish K (2017). Investigation of inhomogeneous and anisotropic material behavior of porcine thoracic aorta using nanoindentation tests. Journal of the Mechanical Behavior of Biomedical Materials 69: 50-56.

Kim J, Baek S (2011). Circumferential variations of mechanical behavior of the porcine thoracic aorta during the inflation test. Journal of Biomechanics 44: 1941-1947.

Manuel R, Bruno B. Verónica D, Mrtín D, German EG, Mrtín AG, Tamara M, Virginia P, Luciana W, Ricardo GJG (2016). Cardiac ischemic preconditioning prevents dystrophin proteolysis by MMP-2 inhibition. Biocell 41: 41-43.

Pandit A, Lu X, Wang C, Kassab GS (2005). Biaxial elastic material properties of porcine coronary media and adventitia. Ajp Heart \& Circulatory Physiology 288: 2581-2587.

Peña JA, Martínez MA, Peña E (2015). Layer-specific residual deformations and uniaxial and biaxial mechanical properties of thoracic porcine aorta. Journal of the Mechanical Behavior of Biomedical Materials 50: 55-69.
Ramanath VS, Oh JK, Iii TMS, Eagle KA (2009). Acute aortic syndromes and thoracic aortic aneurysm. Mayo Clinic Proceedings 84: 465-481.

Raghavan ML, Marshall WW, David AV (1996). Ex vivo biomechanical behavior of abdominal aortic aneurysm: assessment using a new mathematical model. Annals of Biomedical Engineering 24: 573-582.

Roveri N, Ripamonti A, Pulga C, Jeronimidis G, Purslow PP, Volpin D (2010). Mechanical behaviour of aortic tissue as a function of collagen orientation. Macromolecular Chemistry \& Physics. 181: 1999-2007.

Sokolis DP (2007): Passive mechanical properties and structure of the aorta: Segmental analysis. Acta Physiologica 190: 277-289.

Sokolis DP, Boudoulas H, Karayannacos PE (2008). Segmental differences of aortic function and composition: Clinical implications. Hellenic Journal of Cardiology 49: 145-154.

Sokolis DP, Kritharis EP, Giagini AT, Lampropoulos KM, Papadodima SA, Iliopoulos DC (2012). Biomechanical response of ascending thoracic aortic aneurysms: association with structural remodelling. Computer Methods in Biomechanics \& Biomedical Engineering 15: 231-248.

Sokolis DP (2015). Effects of aneurysm on the directional, regional, and layer distribution of residual strains in ascending thoracic aorta. Journal of the Mechanical Behavior of Biomedical Materials 46: 229-243.

Shadwick RE (1999). Mechanical design in arteries. Journal of Experimental Biology 202: 3305-3313.

Shcherbakova D, Papadacci C, Swillens A, Caenen A, Bock SD, Saey V (2014). Supersonic shear wave imaging to assess arterial nonlinear behavior and anisotropy: Proof of principle via ex vivo testing of the horse aorta. Advances in Mechanical Engineering 2014: 1-12.

Tang DL, Li ZY (2016). Preface: computational and experimental methods for biological research: cardiovascular diseases and beyond. Biomedical Engineering Online 15: 219-222.

Tang D, Zuo H, Yang C (2016). Comparison of Right Ventricle Morphological and Mechanical Characteristics for Healthy and Patients with Tetralogy of Fallot: An In Vivo MRI-Based Modeling Study. Mcb Molecular \& Cellular Biomechanics 14: 137-151.

Tang D, Li ZY (2018). Preface: innovations and current trends in computational cardiovascular modeling and beyond: molecular, cellular, tissue and organ biomechanics with clinical applications. Computer Modeling in Engineering \& Science, 116:109-113.

Thubrikar MJ, Labrosse M, Robicsek F, Al-Soudi J, Fowler B (2001). Mechanical properties of abdominal aortic aneurysm wall. Journal of Medical Engineering \& Technology 25: 133-142.

Weisbecker H, Viertler C, Pierce DM, Holzapfel GA (2013). The role of elastin and collagen in the softening behavior of the human thoracic aortic media. Journal of Biomechanics 46: 1859-1865.

Weisbecker H, Unterberger MJ, Holzapfel GA (2015). Constitutive modelling of arteries considering fibre recruitment and three-dimensional fibre distribution. Journal of the Royal Society Interface 12: 1-10.

Wang C, Garcia M, Lu X, Lanir Y, Kassab GS (2006). Threedimensional mechanical properties of porcine coronary arteries: a validated two-layer model. American Journal of Physiology-Heart and Circulatory Physiolog 291: 1200-1209.

Yanxia W, Yu W, Siqi L, Aziz RA, Shutian L, Kairong Q (2018). The Analysis of Wall Shear Stress Modulated by Acute Exercise in the Human Common Carotid Artery with an Elastic Tube Model. Computer Modeling in Engineering \& Science, 116:127-147. 